\title{
Perfil dos casos novos de tuberculose notificados em Ribeirão Preto (SP) no período de 2000 a 2006
}

\author{
Profile of new cases of tuberculosis \\ in Ribeirão Preto, São Paulo State, in the period of 2000 to 2006
}

Paula Hino ${ }^{1}$

Tarcísio N eves da Cunha ${ }^{2}$

Tereza Cristina Scatena Villa ${ }^{3}$

Claudia Benedita dos Santos ${ }^{3}$

${ }^{1}$ Escola de Enfermagem, Universidade de São Paulo. Av. Dr. Enéas de Carvalho Aguiar 419, Bela Vista. 05403-000 São Paulo SP. paulahino@yahoo.com.br ${ }^{2}$ Organização PanAmericanada Saúde ${ }^{3}$ Departamento de Enfermagem $M$ aternoInfantil eSaúdePública, Escola de Enfermagem de Ribeirão Preto,

Universidade de São Paulo.
Abstract The World Health Organization estimates that one third of the world population is infected by the Mycobacterium tuberculosis, while 8-9 million of people develop the disease and 2 million die each year due to tuberculosis, especially in countries and regions with precarious socioeconomic conditions. This study describes the profile of new cases of Tuberculosis notified in Ribeirão Preto, São Paulo State, in the period between 2000 and 2006. A cohort descriptive design was used. The database Epi-Tb was used for thedata collection. Theclinical form pre dominant was pulmonary. It was verified that $71.7 \%$ of patients were male and young adults (20-39 years). Regarding the type of supervision, it was observed that the supervised form presented a progressive increase along the period considered, with exception of 2003. The Tb/HIV coinfection was superior to $25 \%$, while $12.9 \%$ of the patients performed the test. Regarding the treatment result, cure varied from $62.7 \%$ to $76.7 \%$, the abandonment mean was $4.3 \%$ and death by tuberculosis was $3.9 \%$. The results presented contribute for the knowledge of the tuberculosis epidemiological situation along the years studied, providing support to decision making related to prevention actions and control of the disease in the municipality.

Key words Tuberculosis, Epidemiology, Tb/HIV
Resumo A Organização M undial de Saúde estima queum terço da população mundial encontrase infectada pelo Mycobacterium tuberculosis, sendo que 8-9 milhões de pessoas desenvolvem a doença e, anualmente, 2 milhões vão a óbito por tuberculose, principalmenteem paíseseregiões com condições socioeconômicas precárias. Este estudo descreve o perfil dos casos novos de tuberculose notificados no município de Ribeirão Preto (SP) no período de 2000 a 2006. Utilizou-se um dese nho descritivo do tipo coorte. Para a coleta dos dados, foi utilizado o banco de dados Epi-Tb. A forma clínica predominante foi a pulmonar. Verificou-seque $71,7 \%$ dos doentes eram do sexo masculino e adultos jovens (20-39 anos). Em relação ao tipo de supervisão, observou-se aumento gradativo da forma supervisionada ao longo do período considerado, exceto no ano de 2003. A coinfecção T b/HIV mostrou-sesuperior a $25 \%$, sendo que $12,9 \%$ dos doentes não realizaram o teste antiHIV. Q uanto ao resultado de tratamento, a cura variou de $62,7 \%$ a $76,7 \%$, a média do abandono foi de $4,3 \%$ eóbito por tuberculose foi de $3,9 \%$. Os resultados apresentados contribuem para o conhecimento da situação epidemiológi ca da tuberculoseao longo dos anos, fornecendo subsídios à tomada dedecisões relacionadas principalmenteàsações de prevenção e controle da doença no município. Palavras-chave Tuberculose, Epidemiologia, Tb/ HIV 


\section{Introdução}

Recentemente, a tuberculose ( $\mathrm{Tb}$ ) tornou-seuma doença reemergente nos países desenvolvidos, mas manteve a ocorrência em níveis elevados e eventualmentecrescentenos países em desenvolvimento ${ }^{1,2}$. De acordo com Ruffino-N etto ${ }^{3}$, a Tb no Brasil não é um problema de saúde pública emergentenem reemergente e sim um problema "presenteeficante" hálongo tempo. Nasúltimas décadas, o controle da Tb foi grandementenegligenciado pelas políticas públicas, sociedadee comunidade científica, na ilusão de que o problema estaria sob controle ou resolvido.

0 problema da Tb no Brasil reflete o estágio de desenvolvimento social do país, onde os determinantes do estado de pobreza, as condições sanitárias precárias, as fraquezas de organização do sistema de saúde e as deficiências de gestão limitam a ação da tecnologia e, por consequência, inibem a queda de doenças marcadas pelo contexto social.

Com a edição da Norma O peracional Assistência à Saúde (NOAS/2001), a Tb foi incorporada ao Departamento de Atenção Básica; a partir deste momento, tanto os municípios em Gestão Plena da Atenção Básica quanto os queseencontram em Gestão Plena do Sistema M unicipal devem se organizar para atender o doente de Tb.

Em 2006, a Tb volta a ser firmada como prioridade de saúde pública pelo Pacto pela Vida, que firma o compromisso acerca das prioridades que apresentam impacto na situação de saúde da população. Cabe aos municípios, estadose regiões planejar as ações necessárias de acordo com cada realidade local para que as metas e objetivos delineados sejam atingidos. Uma das metas para o controle da Tb aponta para a necessidade de se atingir pelo menos $85 \%$ de cura dos casos novos de Tb bacilífero diagnosticados a cada ano ${ }^{4}$.

A presente investigação teve como objetivo descrever o perfil dos casos novos da Tb no município de Ribeirão Preto (SP) no período de 2000 a 2006.

\section{M etodologia}

Este é um estudo descritivo de coorte. Foram coletadas informações do banco de dados Epi$\mathrm{Tb}$, referente aos casos novos de $\mathrm{Tb}$ notificados no período de 2000 a 2006, residentes em Ribeirão Preto. Para a realização do referido estudo, 0 projeto foi aprovado pelo Comitê de Ética em
Pesquisa da Escola de Enfermagem de Ribeirão Preto da Universidade de São Paulo. Como este estudo utilizou exclusivamente informações das fichas de notificação compulsória da Tb e este não envolveu a identificação dos doentes, foi solicitada a dispensa do termo de consentimento livre e esclarecido devido à garantia do si gilo.

\section{Resultados}

No município de Ribeirão Preto, foram notificados no período considerado um total de 1.170 casos novos de $\mathrm{Tb}$, distribuídos ao longo do período considerado neste estudo conforme ilustrado na Tabela 1, juntamente com seus respectivos coeficientes deincidência.

Em relação à forma clínica, observou-se pre domínio da forma pulmonar. A forma pulmonar manteve-se estável para todos os anos, variando de $72 \%$ (2002) a $78 \%$ (2006).

Quanto ao gênero dos doentes, verificou-se que $71,7 \%$ eram do sexo masculino, predominando sobre o sexo feminino para todos os anos do estudo.

A Tabela 2 apresenta a distribuição dos casos novos de Tb segundo faixa etária (anos). Houve onze perdas deinformação; portanto, trabalhouse com 1.159 casos. Em relação aos menores de quinze anos, houve predomínio em menores de cinco anos (44\%) e variação entre o período estudado de $0,5 \%$ (2001) a 3,1\% (2002). A maior proporção dos casos de Tb em Ribeirão Preto ocorreu na faixa etária de vinte a 49 anos, com ênfase no grupo de doentes de trinta a 39 anos (27,9\%). A ocorrência da doença no grupo de

Tabela 1. Coeficientes de incidência de tuberculose por 100.000 habitantes. Ribeirão Preto (SP), 2000 a 2006.

\begin{tabular}{ccc}
\hline Ano & $\begin{array}{c}\text { Casos } \\
\text { novos }\end{array}$ & $\begin{array}{r}\text { Coeficiente de incidência } \\
\text { (/100.000 habitantes) }\end{array}$ \\
\hline 2000 & 183 & 36,2 \\
2001 & 200 & 38,9 \\
2002 & 161 & 30,9 \\
2003 & 163 & 30,9 \\
2004 & 164 & 30,7 \\
2005 & 149 & 27 \\
2006 & 150 & 26,8 \\
\hline
\end{tabular}

Fonte: Epi-Tb; População: IBGE 
Tabela 2. Distribuição dos casos novos de tuberculose segundo faixa etária. Ribeirão Preto (SP), 2000-2006.

\begin{tabular}{rrrrrrrrrrrrr}
\hline \multicolumn{10}{c}{ Faixa etária (anos) } \\
\cline { 2 - 6 } & 0 a 4 & 5 a 9 & 10 a 14 & 15 a 19 & 20 a 29 & 30 a 39 & 40 a 49 & 50 a 59 & 60 ou + $\begin{array}{c}\text { Sem } \\
\text { informação }\end{array}$ \\
\hline 2000 & 4 & 1 & 3 & 5 & 35 & 54 & 41 & 20 & 19 & 1 & 183 \\
2001 & 1 & 2 & 0 & 0 & 33 & 58 & 56 & 28 & 20 & 2 & 200 \\
2002 & 5 & 2 & 2 & 3 & 29 & 44 & 30 & 30 & 16 & 0 & 161 \\
2003 & 2 & 2 & 2 & 10 & 32 & 33 & 33 & 21 & 25 & 3 & 167 \\
2004 & 3 & 2 & 2 & 6 & 18 & 53 & 34 & 25 & 17 & 4 & 164 \\
2005 & 1 & 2 & 1 & 9 & 23 & 38 & 26 & 21 & 27 & 1 & 149 \\
2006 & 4 & 3 & 1 & 6 & 27 & 43 & 28 & 16 & 22 & 0 & 150 \\
Total & 20 & 14 & 11 & 39 & 197 & 323 & 248 & 161 & 146 & 11 & 1170 \\
\hline
\end{tabular}

adultose, supostamente, pessoas economicamenteativas pode ser explicada pelos hábitos de vida, aumento da prevalência do HIV, alcoolismo, entre outros.

A média das idades oscilou entre 38,9 anos (2000) e 42 anos (2005) e a mediana entre 37 anos (2000 e 2006) e 40 anos (2001), sendo as idades mínima e máxima iguais a zero e 96 anos, respectivamente.

Importante destacar a presença de $12,6 \%$ dos doentes acima de sessenta anos, sendo que este percentual variou ao longo do período estudado de $9 \%$ (2002) a 18,2\% (2005).

A porcentagem dos casos novos de $\mathrm{Tb}$ que receberam a medicação antituberculose de forma supervisionada foi de $41 \%$ no ano 2000 , alcançando $76,7 \%$ em 2006. N otou-se um aumento gradual da cobertura do tratamento supervisionado (TS), exceto ao observado no ano de 2003 , quando a queda foi de $7,8 \%$ referente ao ano anterior.

Em relação à coinfecção $\mathrm{Tb} / \mathrm{HIV}$, notou-se um padrão elevado e estável (superior a $25 \%$ ) para o período considerado neste estudo, exceto no ano de2005, quando o percentual mínimo foi de $18 \%$, que pode ser atribuído ao fato da não realização do teste em $16,1 \%$ dos doentes. Apesar do teste anti-HIV ser recomendado para todos os casos diagnosticados de $T b$, devido à alta prevalência da coinfecção Tb/HIV, observou-se que estenão foi realizado em $12,9 \%$ dos doentes.

Em Ribeirão Preto, observou-se que muitos doentes que apresentaram a coinfecção Tb/HIV foram a óbito antes de concluir o tratamento da $\mathrm{Tb}$, sendo que a porcentagem de óbitos não relacionados à Tb variou de $26,4 \%$ a 49\%. 0 percentual de doentes que foram a óbito durante o tra- tamento da $\mathrm{Tb}$ e que tiveram a $\mathrm{Tb}$ como causa básica na declaração de óbito variou de 0 a 1,9\%.

A Tabela 3 retrata o resultado de tratamento dos casos novos de Tb para os casos notificados no período de 2000 a 2006 em Ribeirão Preto. Em relação à cura, esta foi de $74,3 \%$ no ano de 2000, mostrando um pequeno decréscimo para 0 ano seguinte, voltando a aumentar nos anos de 2002 e2003. N ota-seum período de oscilação entre 2004 e 2006, quando, neste último ano, 0 percentual foi de $62,7 \%$, considerado abaixo da meta preconizada pelo Programa $\mathrm{Nacional}$ de Controleda Tuberculose, queédecurar $85 \%$ dos casos. 0 abandono em Ribeirão Preto vem se apresentando em declínio desde 1996, visto que nesta época o percentual era de $19 \%$. O bservouse para o período estudado um percentual que variou de $2,7 \%$ (2000) a 6,2\% (2002). Os óbitos que tiveram a Tb como causa básica variaram de $1,3 \%$ (2006) a 6,1\% (2005) e os óbitos dos doentes de Tb que tiveram outras causas de óbito que não $a T b$ apresentaram um comportamento mais irregular; a médiafoi de $13,4 \%$, variando de $9,5 \%$ (2005) a 21,7\% (2004).

A Tabela 4 apresenta os casos novos de Tb soropositivos ao HIV segundo encerramento para os casos notificados no período de 2000 a 2006 em Ribeirão Preto. A cura dos doentes que apresentaram coinfecção $\mathrm{Tb} / \mathrm{HIV}$ apresentou variação de 37,2\% (2006) a 60,4\% (2000), percentuais considerados baixos e que podem ser atribuídos à elevada taxa de abandono e óbito. Quanto ao abandono, as porcentagens observadas nos anos de 2001 e 2005 foram as mais elevadas, 14\% e $14,3 \%$,respectivamente. No ano de 2006 , até 0 momento da coleta dos dados, não havia sido informado nenhum caso de abandono. 
Tabela 3. Casos novos de tuberculose (\%) segundo resultado de tratamento. Ribeirão Preto (SP), 2000-2006.

\begin{tabular}{ccccccc}
\hline & \multicolumn{7}{c}{ Encerramento } \\
\cline { 2 - 7 } Ano & $\begin{array}{c}\text { Cura } \\
(\%)\end{array}$ & $\begin{array}{c}\text { Abandono } \\
(\%)\end{array}$ & $\begin{array}{c}\text { Transferência } \\
(\%)\end{array}$ & $\begin{array}{c}\text { Mudança } \\
\text { diagnóstico }(\%)\end{array}$ & $\begin{array}{c}\text { Óbito } \\
\text { Tb }(\%)\end{array}$ & $\begin{array}{c}\text { Óbito não } \\
\text { Tb }(\%)\end{array}$ \\
\hline 2000 & 74,3 & 2,7 & 3,8 & 3,8 & 4,9 & 10,4 \\
2001 & 73 & 6 & 2,5 & 2 & 3 & 13,5 \\
2002 & 77 & 6,2 & 1,2 & 2,5 & 2,5 & 10,6 \\
2003 & 76,7 & 3,7 & 0,6 & 0 & 3,7 & 15,3 \\
2004 & 66,5 & 5 & 1,2 & 0 & 5,6 & 21,7 \\
2005 & 76,4 & 4,1 & 4,1 & 0 & 6,1 & 9,5 \\
2006 & 62,7 & 2,7 & 5,3 & 10 & 1,3 & 12,7 \\
Total & 72,4 & 4,3 & 2,7 & 2,6 & 3,9 & 13,4 \\
\hline
\end{tabular}

Tabela 4. Doentes com coinfecção Tb/HIV segundo resultado de tratamento. Ribeirão Preto (SP), 2000-2006.

\begin{tabular}{rrrrrrr}
\hline Ano & $\begin{array}{c}\text { Cura } \\
(\%)\end{array}$ & $\begin{array}{c}\text { Abandono } \\
(\%)\end{array}$ & $\begin{array}{c}\text { Transferência } \\
(\%)\end{array}$ & $\begin{array}{c}\text { Mudança } \\
\text { diagnóstico (\%) }\end{array}$ & $\begin{array}{r}\text { Óbito } \\
\text { Tb (\%) }\end{array}$ & $\begin{array}{r}\text { Óbito não } \\
\text { Tb (\%) }\end{array}$ \\
\hline 2000 & 60,4 & 5,7 & 5,7 & 0 & 1,9 & 26,4 \\
2001 & 40 & 14 & 2 & 6 & 0 & 38 \\
2002 & 58,1 & 9,3 & 2,3 & 2,3 & 0 & 27,9 \\
2003 & 53,5 & 4,7 & 0 & 0 & 0 & 41,9 \\
2004 & 43,1 & 7,8 & 0 & 0 & 0 & 49 \\
2005 & 46,4 & 14,3 & 7,1 & 0 & 0 & 32,1 \\
2006 & 37,2 & 0 & 11,6 & 23,2 & 0 & 27,9 \\
Total & 48,4 & 7,7 & 3,8 & 4,5 & 0,3 & 35 \\
\hline
\end{tabular}

\section{Discussão}

0 quadro registrado em Ribei rão Preto demonstra o paradoxo de um município que, apesar de abrigar um dos maiores centros de excelência médica do país, apresenta ainda valores elevados de incidência de $T b$, sendo, portanto, considerado prioritário para o controle da doença. A elevada associação com o HIV, em torno de $30 \%$, agrava ainda mais a situação da doença, prejudicando o percentual de cura eelevando 0 abandono e a mortalidade.

A caracterização dos doentes de acordo com a forma clínica mostrou predomínio da forma pulmonar (74,5\%), não diferindo da distribuição encontrada nos estudos epidemiológicos em geral $\left.\right|^{5-8}$.

Importante ressaltar que a forma pulmonar é a que merece maior atenção para as atividades de controle da Tb por ser a forma transmissível da doença. Daí a necessidade do diagnóstico e início do tratamento da Tb o mais rápido possível, como medidas de intervenção na cadeia de transmissão da doença. Sendo a forma pulmonar defácil diagnóstico e de baixo custo, esperase que o mesmo possa ser realizado no nível primário de atenção.

Em relação ao sexo dos doentes de $T b$ em Ribei rão Preto, o predomínio do sexo masculino mostrou concordância com o observado na literatura-12.

Em Ribeirão Preto, os maiores coeficientes de incidência debacilíferosforam observadosna faixa etária de vintea 49 anos, com ênfase nos doentes de trinta a 39 anos. Tal resultado vem ao encontro da literatura ${ }^{5,7}$, mostrando que a Tb acomete mais a classe de adultos jovens, que representa 0 setor mais produtivo da população. Esta situação gera complicações sociais importantes, considerando que este grupo deveria estar inserido no mercado de trabalho e provendo o sustento das famílias e que, muitas vezes, devido à debili- 
dade física que pode se encontrar graças à doença, fica impossibilitado de trabalhar.

0 predomínio de $12,6 \%$ dos doentes na acima de sessenta anos ressalta que $\mathrm{T} \mathrm{Tb}$ mereceum enfoque nesta faixa etária, visto que vem sendo responsável por um percentual significativo nas estatísticas oficiais, cabendo alertar os profissionais de saúde para que estejam atentos quanto à possibilidade da ocorrência da Tb nesta população, a fim de diminuir os danos e óbitos. Esta situação pode ser explicada pelo crescimento da população idosa como consequência do aumento da expectativa de vida. A população de idosos em Ribeirão Preto, que no ano de 2000 era de 51.194 habitantes, aumentou para 56.743 habitantes em 2006.

Nos países desenvolvidos, a situação encontrada é diferente; observa-se um acometimento maior da Tb na população idosa, decorrente da exposição que sofreu na infância e, tendo sido infectada pelo bacilo, pode vir a desenvolver a doença devido às deficiências próprias do enveIhecimento. Acrescido a isto, outros fatores contribuem para acometer esta faixa etária, como dificuldade de acesso aos serviços de saúde, idosos confinados em asilos, demora na procura da assistência médica, devido à pouca importância dada aos sintomas por acharem ser próprios de doenças que acometem os idosos ${ }^{13}$.

Em relação ao TS, Ribeirão Preto foi o primeiro município no Brasil a atender as recomendações do M inistério da Saúde (M S) em relação à supervisão do tratamento da $\mathrm{Tb}^{14}$. 0 trabalho iniciou-seem setembro de 1997 em uma unidade de referência de tratamento da Tb situada na região sul do município, tendo sua expansão para outras unidades no ano seguinte.

A cobertura do TS no seu ano de implantação (1998) foi de $19,5 \%$, aumentando para $29,9 \%$ no ano seguinte ${ }^{15}$. Inicialmente, priorizavam-se os doentesqueofereciam risco deabandono, taiscomo al coólatras, usuários de drogas, HIV positivo, caso de abandono anterior ou recidiva, entre outros. Ao longo do tempo, esta oferta vem seestendendo a todos os casos pulmonares bacilíferos.

A estratégia do TS desponta no espaço da saúde como um novo modo de agir em saúde, capaz de gerar mudanças na prática dos profissionais envolvidos ${ }^{14}$. Privilegia ações educativas no âmbito familiar, ampliando a capacidade de atuação dos agentes através do estabelecimento de vínculo, acolhimento, na perspectiva de garantir maior qualidade da atenção e adesão do doente ao tratamento, além de melhorar osindicadores de cura e reduzir o abandono e interferir na transmissão, impedindo a resistência aos medicamentos.

Apesar da estratégia Directly Observed The rapy (DOTS) ser considerada essencial para 0 controle da $\mathrm{Tb}$, os cinco componentes sozinhos (compromisso político, detecção de casos por baciloscopia, supervisão medicamentosa, suprimento regular de medicamentos e sistema de informação) não são suficientes para reverter a situação da Tb. São necessários também o fortale cimento dos sistemas de saúde, o envolvimento dos profissionais de saúdee da comunidade, atenção especial aos casos de coinfecção e multidrogarresistência, bem como o incentivo à pesquisa voltada ao desenvolvimento de novas tecnologias para o efetivo controle da doença eal cance das metas pactuadas ${ }^{15}$.

0 resultado encontrado de elevada coinfecção Tb/HIV registraa importânciaqueestas duas doenças têm no município; isso podeser explicado através da análise da incidência da aids no estado de São Paulo, que demonstrou o fato de que regiões mais populosas apresentam maiores riscos de transmissão da $\mathrm{Tb}$, como a capital do estado e municípios como Santos, São José do Rio Preto e Ribeirão Preto ${ }^{16}$.

0 fato do teste anti-HIV não ter sido realizado em um grupo significativo neste estudo $(12,9 \%)$ demonstra a existência defalhas nos serviços de saúde, além de impedir o conhecimento da real situação da coinfecção Tb/HIV em Ribeirão Preto. Tal situação pode ser explicada por autores ${ }^{17}$ que acreditam que esta situação ocorre porque 0 teste para se determinar o HIV pode ser considerado discriminatório por parte do doente, pois apesar da sua realização ser uma recomendação do MS, para realizá-lo é necessária a autorização do doente, que pode recusá-lo por direito de cidadania.

Este fato é preocupante, pois muitos dos indivíduos que apresentam Tb podem também ser portadores do HIV, além de apresentar resultados mais desfavoráveis em relação ao resultado detratamento quando comparados ao grupo dos doentes não coinfectados.

0 conhecimento do percentual de coinfecção Tb/HIV faz-se necessário para que seja possível realizar um planejamento das ações de controle da Tb mais adequado a esta clientela, como por exemplo, adotar medidas terapêuticas adequadas que evitem a resistência aos tuberculostáticos, maior rigor no uso de medicamentos para evitar o abandono do tratamento e/ ou tratamento irregular. Devido à al ta prevalência da coinfecção Tb/HIV no município, estas duas doenças 
não podem ser discutidas isoladamente; portanto, os profissionais de saúde devem estar atentos tanto em relação ao diagnóstico precoce da infecção pelo HIV, quanto ao doente de Tb.

Quanto à variável resultado de tratamento, embora o percentual de cura em Ribeirão Preto ainda não tenha alcançado a meta proposta pelo MS, que é de curar $85 \%$ dos casos diagnosticados, observou-semelhora dosindicadores, quando se considera que em 1995 este percentual era de $47 \%$.

$\mathrm{N}$ ota-se um percentual de abandono no ano de 2001 de $6 \%$, sendo que, no ano anterior, este percentual era de 2,7\%. Este aumento pode ser atribuído à interrupção das atividades do agente de saúde que realizava o TS dos doentes com coinfecção Tb/HIV da Unidade Especial de Tratamento de Doenças I nfecciosas do H ospital das Clínicas da Faculdade de M edicina de Ribeirão. Tal situação voltou a se normalizar apenas no ano de 2003, apresentando pequena inclinação para 0 ano seguinte.

A porcentagem de abandono em Ribeirão Preto vem obedecendo desde 2003 a meta traçada pelo MS, queé de $5 \%$. No Brasil, este percentual apresenta-se variável nas suas diversas regiões, de 4,5 a $20,3 \%$.

0 abandono do tratamento envolve uma série de fatores e não pode ser atribuída culpabilidade apenas ao doente; devem ser consideradas também questões relacionadas aos serviços de saúde, como a desorganização do trabalho em equipe, demora do atendimento, desumanização, falta de vínculo entre doente e profissional de saúde, ausência de busca ativa ao doente e faltoso, entre outros.

\section{Considerações finais}

Para o período considerado neste estudo, o percentual de óbito foi considerado elevado, visto que $17,3 \%$ dos doentes foram a óbito enquanto realizavam o tratamento da $\mathrm{Tb}$, sendo 3,9\% dos óbitos relacionados à $\mathrm{Tb}$ (causa básica) e 13,4\% tiveram outra causa que não a Tb.

A taxa de mortalidade que teve a $\mathrm{Tb}$ como causa básica de óbito em Ribeirão Preto, mesmo que aparentemente subestimada, exige reflexões em busca de uma explicação, visto que a Tb é uma doença quepodeser prevenida ecurável em quase $100 \%$ dos casos, desde que sejam seguidos o esquema terapêutico adequado e a regularidade da ingestão medicamentosa. Neste caso, o óbito de um indivíduo com Tb deveria ser um evento excepcional.

Atéo momento da coleta de dados, $5,3 \%$ dos casos referentes ao ano de 2006 ainda constavam na situação como "em andamento"; portanto, os valores apresentados para o referido ano estão sujeitos a alterações, embora pequenas.

As estratégias de interven ção e metas a serem alcançadas para a Tb nas últimas décadas são caracterizadas por ações dirigidas à detecção de casos e tratamento dos doentes; observa-se pouca atenção dada ao contexto social de desigualdades em que a população mais exposta está inserida. São necessárias novas estratégias intersetoriais que visem à promoção da saúde e melhoria da qualidade de vida de determinados grupos populacionais.

Portanto, a Tb deve ser orientada por uma visão que vai além das fronteiras da clínica; devem ser envolvidas as condições de vida em que estes doentes estão inseridos, visto que não é recente a discussão de que a pobreza é 0 ambiente natural da Tb. Enquanto considerarmos o controle da Tb apenas como um somatório de doentes que precisam de uma intervenção biomédica, focada no alcance da cura, continuaremos a ter casos de $\mathrm{Tb}$ em nosso meio. 


\section{Colaboradores}

P H ino foi responsável pela coleta e organização do banco de dados, análise/discussão dos dados e redação do artigo. CB Santos e TN Cunha definiram a metodologia do estudo, colaborou no processamento e análise dos dados bem como na revisão final do artigo. TCS Villa trabalhou na revisão final do artigo.

\section{Referências}

1. Floyd K, Pantoja A. Financial resources required for tuberculosis control to achieve global targets set for 2015. Bull World H ealth O rgan 2008; 86(7):568576.

2. Xavier MIM, Barreto ML. Tuberculose na cidade de Salvador, Bahia, Brasil: o perfil na década de 1990. Cad Saude Publica 2007; 23(2):445-453.

3. Ruffino-Netto A. Tuberculose: a calamidade negligenciada. Rev Soc Bras M ed Trop 2002; 35(1):51-58.

4. Brasil. M inistério da Saúde. Secretaria Executiva. Departamento de Apoio à Descentralização. Coordenação-Geral de Apoio à Gestão Descentralizada. Diretrizes operacionais dos Pactos pela Vida, em D efesa do SUS e de Gestão. Brasília: Ministério da Saúde; 2006.

5. Chaimowicz F. Age transition of tuberculosis incidence and mortality in Brazil. Rev. Saude Publica 2001; 35(1):81-87.

6. Small PM, Fujiwara PI. Management of tuberculosis in the United States. New Engl J Med 2001; 345(3):189-200.

7. Paixão LMM, Gontijo ED. Perfil dos casos de tuberculose notificados e fatores associados ao abandono, Belo Horizonte, M G. Rev. Saude Publica 2007; 41(2):205-213.

8. Selig L, Belo M, Cunha AJLA, Teixeira EG, Brito R, Luna $A L$, Trajman $A$. Ó bitos atribuídos à tubercuIose no estado do Rio de Janeiro. J Bras Pneumol 2004; 30(4):417-424.

9. Ferreira SM B, Silva AM C, Botelho C. Abandono do tratamento da tuberculose pulmonar em CuiabáMT-Brasil. J Bras Pneumol 2005; 31(5):427-435.

10. Hiijar MA. Tuberculose: desafio permanente. Cad Saude Publica 2005; 21(2): 348-349.

11. Lima M B, M ello DA, M orais AP, Silva WC. Estudo de casos sobre abandono do tratamento da tuberculose: avaliação do atendimento, percepção e conhecimentos sobre a doença na perspectiva dos clientes (Fortaleza, Ceará, Brasil). Cad Saude Publica $2001 ; 17(4): 877-885$.
12. Oliveira HB, Marin-León L, Cardoso JC. Perfil de mortalidade de pacientes com tuberculose relacionada à comorbidade tuberculose- aids. Rev. Saude Publica 2004; 38(4):503-510.

13. Cavalcanti ZR, Albuquerque M FPM, Campelo ARL, Ximenes RAA, Montarroyos $U$, Verçosa MKA. Característica da Tuberculose em idosos no Recife $(\mathrm{PE})$ : contribuição para o programa de controle. J Bras Pneumol 2006; 32(6):424-429.

14. M uniz JN, Villa TCS, Ruffino-N etto A. A experiência de implantação do DOTS em Ribeirão Preto. In: Villa TCS, Ruffino-N etto A, organizadores. Tuberculose: implantação do DOTS em algumas regiões do Brasil. Histórico e peculiaridades regionais. Ribeirão Preto: Rede-Tb; 2006. p. 116-125.

15. Hino $P$, Santos CB, Villa TCS. Evolução espaçotemporal dos casos de tuberculose em Ribeirão Preto (SP), nos anos de 1998 a 2002. J Bras Pneumol 2005; 31(6):523-527.

16. Rodrigues-JR AL, Ruffino-N etto $A$, Castilho EA. Distribuição espacial da co-infecção M .tuberculosis/ HIV no Estado de São Paulo, 1991-2001. Rev. Saude Publica 2006; 40(2):265-270.

17. Carvalho LGM, Buani AZ, Zollner MSAC, Scherma A. Co-infecção por M ycobacterium tuberculosis e vírus da imunodeficiência humana: uma análise epidemiológica em Taubaté (SP). J Bras Pneumol 2006; 32(5):424-429.

Artigo apresentado em 14/04/2008

Aprovado em 15/08/2008

Versão final apresentada em 20/09/2008 